\title{
Detection and Correction of Doppler Outliers in Kalman Filter-based Positioning*
}

\author{
Atsushi Mouri ${ }^{\dagger \ddagger}$, Yukihiro Kubo ${ }^{\ddagger}$, Sueo Sugimoto ${ }^{\ddagger}$ and Masaharu OHASHI ${ }^{\S}$
}

\begin{abstract}
In this paper, we propose methods of detecting Doppler outliers which cause positioning errors at Doppler-aided GNSS (Global Navigation Satellite System) positioning, and correcting the errors. We apply the existing detection method based on the innovation process in Kalman filtering to Doppler outlier problems, and we propose a novel detection method based on the measurements by the difference between $\mathrm{C} / \mathrm{A}$ code pseudoranges and Doppler shift range-rates. Both methods are based on chi-squared tests. We apply two correction methods which are Doppler bias exclusion, or the estimation for detected anomalies. The efficient detection of anomalous observables can be developed to RAIM (Receiver Autonomous Integrity Monitoring), and useful to achieve higher accuracy positioning for increasing satellite signals by multi-frequencies and multi-GNSSs. Doppler shift observables are utilized on a priority basis even in urban areas because of immunity to cycleslip and continuous availability, however unexpected Doppler outliers prone to cause positioning errors. The experimental results of positioning by using real receiver data show the feasibility of the proposed detection and correction methods.
\end{abstract}

\section{Introduction}

In this paper, we present methods of detecting and correcting Doppler outliers which cause positioning errors in Doppler-aided Kalman filter-based GNSS (Global Navigation Satellite System) positioning.

In general, low cost single frequency GNSS (Global Navigation Satellite System) receivers equipped on vehicles, mobile phones and so on mainly utilize the so-called $\mathrm{C} / \mathrm{A}$ code pseudorange which is the measured distance between the satellite and the receiver based on the signal travelling time[1]. In this paper, we focus on the position accuracy improvement with the low cost single frequency receiver.

The $\mathrm{C} / \mathrm{A}$ code pseudorange is widely utilized for positioning, however it is well known that it can be

\footnotetext{
* Manuscript Received Date: June 29, 2015

This paper has already been presented in Proc. of the 46th ISCIE International Symposium on Stochastic Systems Theory and Its Applications (SSS'14) which was held in Nov. 1-2, 2014.

† Sanda works, Mitsubishi Electric Corporation; 2-3-33, Miwa, Sanda-city, Hyogo 669-1513, JAPAN

₹ Faculty of Science and Engineering, Ritsumeikan University; Noji-Higashi, Kusatsu-city, Shiga 525-8577, JAPAN

$\S$ Department of Electronic System Engineering, The University of Shiga Prefecture; 2500 Hassaka-cho, Hikone-city, Shiga 525-8533, JAPAN

Key Words: GNSS positioning, Doppler outliers, Kalman filter positioning.
}

easily deteriorated by noises and/or multipath effects on the signal path from the satellite to the receiver, and the position estimate may be largely degraded $[2,3]$.

In order to improve the accuracy of the $\mathrm{C} / \mathrm{A}$ code pseudorange as well as the positioning, the carriersmoothed pseudorange has been proposed [4]. The carrier-smoothed pseudorange is the measurement which is obtained by combining the pseudorange and the low-noise carrier phase observable. The carrier phase observable is obtained by counting the carrier-phase numbers. It is reported that the positioning accuracy can be improved by using the carrier-smoothed pseudorange [4].

However, in general, the carrier phase measurement is available only with high grade, high cost multi frequency receivers for surveyors, geophysical researchers, etc., and such receivers can not be applied for the civilian navigation. Therefore, alternative to the carrier phase measurement, the application of the Doppler shift measurement has been proposed as the so-called Doppler-aided positioning [5,6]. The Doppler frequency shift is caused by the relative motion of the satellite and the receiver, in other words, the variation of the distance between the satellite and the receiver, and it can be measured by the low cost receiver. In $[5,6]$, the Doppler-smoothed pseudorange is introduced based on the similar technique of the carrier-smoothed pseudorange as the preprocessing of the position calculation, and the improvement of the accuracy is reported. In our previous work [7], the Doppler measurement is also utilized for the precise positioning 
using high cost receiver, and the applicability of the Doppler measurement is reported. The positioning algorithm in [7] is based on the multi frequency pseudorange, Doppler and carrier phase measurements, and the Kalman filter is applied to estimate the vehicle position, velocity and acceleration with the appropriate dynamics model of the vehicle. The algorithm can be easily modified to use the single frequency pseudorange and Doppler measurement of the low cost receiver.

By using the modified algorithm of [7], the test drives were mainly conducted in Japan and the United States in 2014. The results show that the Doppler shift measurement sometimes has a bias error due to low signal level or multipath effects. In such case, the positioning algorithms which utilize the Doppler measurement have large positioning errors. Actually we had a vehicle positioning error in real test drives under open sky environment [8]. Therefore, to keep the positioning accuracy high, it is important to detect and correct or exclude the outlier of the Doppler measurement.

Although there are a lot of literature about detection of the outlier or the robust Kalman filter $[9,10]$ to overcome these problems, we propose the following two methods: 1) statistical tests on the innovation processes of the Kalman filter, 2) statistical test on the difference between the variation of the $\mathrm{C} / \mathrm{A}$ code pseudorange and the Doppler shift measurement. Method 1 is derived by modifying the cycle slip detection algorithm in $[11,12]$. Method 2 is the novel method proposed in this paper.

This paper is organized as follows. Section 2 shows the advantages of Doppler shift frequency measurement utilized at low cost single frequency receivers. Section 3 and 4 show the review of the positioning algorithm [7] and the modified GR (GNSS Regression) models for low cost GNSS receivers which utilize code pseudoranges and Doppler observables. Section 5 shows that the two detection methods for Doppler outliers. Section 6 shows that the two correction methods for the impacts caused by Doppler outliers. Section 7 shows that the experimental results by the combinations of the detections and the corrections. Finally the concluding remarks are given in section 8 .

\section{Advantages of Doppler Measurement}

L1-C/A code pseudoranges and Doppler shift frequencies are measured at a satellite signal acquisition stage of GNSS receivers. Receivers conduct a search process over the space of code phase shifts and Doppler-frequency shifts, and routinely measure them in the carrier tracking loop of an acquisition stage. Doppler observables are obtained by FLL (Frequency Locked Loop) and less affected by noises or multipath compared with $\mathrm{C} / \mathrm{A}$ code pseudorange observables obtained by DLL (Delay Locked Loop), and more ro-

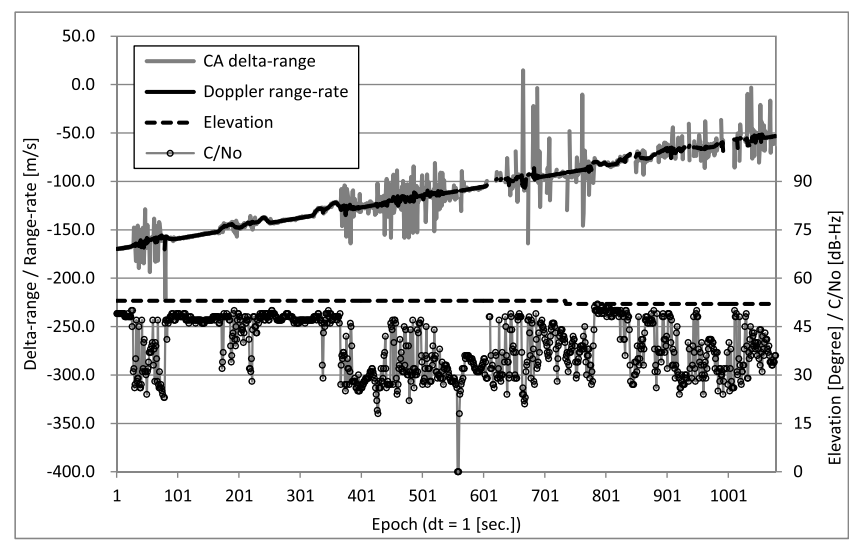

Fig. 1 CA code delta-range / Doppler shift range-rate

bust than carrier-phase observables obtained by PLL (Phase Locked Loop) [13]. We analyze Doppler frequency shift accuracy compared with L1-C/A code pseudoranges. C/A code delta-ranges are the timedifferenced pseudorange observables over two consecutive times (epochs), namely $\rho_{C A, u}^{p}(t)-\rho_{C A, u}^{p}(t-$ $1)$. On the other hand, Doppler shift range-rates are receiver-satellite values based on Doppler frequency shift observables, namely $\lambda_{1} D_{L 1, u}^{p}(t) . \quad \rho_{C A, u}^{p}(t)$ is a $\mathrm{C} / \mathrm{A}$ code pseudorange, and $D_{L 1, u}^{p}(t)$ is a Doppler frequency shift between satellite $p$ and receiver $u$ at epoch $t . \lambda_{1}$ is wave length of carrier wave and calculated by $c / f_{1} . c\left(\approx 2.99792458 \times 10^{8}[\mathrm{~m} / \mathrm{s}]\right)$ denotes the speed of light, and $f_{1}$ is L1 central frequency, namely $f_{1}=2 \times 77 \times 10.23[\mathrm{MHz}]=1575.42[\mathrm{MHz}]$. In theory delta-ranges are nearly equal to range-rates. $\mathrm{C} / \mathrm{A}$ code pseudoranges and Doppler shifts observables are proved to be independent (p.186 in [14]).

Fig. 1 shows an example of the comparison between the delta-ranges (gray line) obtained from code pseudoranges and the range-rates (black line) obtained from Doppler shifts. The observables was collected from a u-blox NEO-7N receiver equipped in the test vehicle moved in Tokyo, Japan, on April 2, 2014. The satellite elevation is 55-56 degrees (dotted line), and the C/No (Carrier to Noise ratio) values (p.117 in [1]) (line with circle) calculated by the baseband process of the receiver are degraded to about 30 from $45 \mathrm{~dB}-$ $\mathrm{Hz}$ because of multipath indirect waves. The deltaranges have several tens of meter fluctuations when the $\mathrm{C} /$ No degradations. On the other hand the rangerates have small fluctuations. The Doppler shifts are less affected by the indirect waves, and can stay more accurate than code pseudoranges even under multipath environments. Therefore Doppler observables have the potential to be utilized to smooth the code pseudorange noises in GNSS difficult environment.

\section{GR Models for Doppler-based Kalman Filter Positioning}

We have already developed the algorithms based on GR models for precise positionings [7]. We formulate the Kalman filter-based positioning by extract- 
ing the equations related to code pseudoranges and Doppler shift observables from the GR models. The natural extensions of GNSS regression models for multiple frequencies of various GNSS modernizations are also similarly formulated.

We consider the following fundamental observables of the pseudoranges $\rho_{C A, u}^{p}(t)$ based on $\mathrm{C} / \mathrm{A}$ codes, and the range-rates $\dot{\rho}_{D L 1, u}^{p}(t)$ based on Doppler shift frequencies as follows:

$$
\begin{aligned}
\rho_{C A, u}^{p}(t)= & r_{u}^{p}\left(t, t-\tau_{u}^{p}\right)+\delta I_{u}^{p}(t)+\delta T_{u}^{p}(t) \\
& +c\left[\delta t_{u}(t)-\delta t^{p}\left(t-\tau_{u}^{p}\right)\right] \\
& +B_{C A, u}^{p}(t)+e_{C A, u}^{p}(t), \\
\dot{\rho}_{D L 1, u}^{p}(t)= & \dot{r}_{u}^{p}\left(t, t-\tau_{u}^{p}\right)+\delta \dot{I}_{u}^{p}(t)+\delta \dot{T}_{u}^{p}(t) \\
& +c\left[\delta \dot{t}_{u}(t)-\delta \dot{t}^{p}\left(t-\tau_{u}^{p}\right)\right] \\
& +b_{D L 1, u}^{p}(t)+\varepsilon_{D L 1, u}^{p}(t),
\end{aligned}
$$

where in equation $(1), r_{u}^{p}\left(t, t-\tau_{u}^{p}\right)$ is the geometric distance between the receiver $u$ at the time $t$ and the satellite $p$ at the time $t-\tau_{u}^{p}\left(\tau_{u}^{p}\right.$ denotes the signal travel time from the satellite $p\left(p=1, \ldots, n_{s}\right)$ to the receiver $u$ ). Namely,

$$
\begin{aligned}
r_{u}^{p}(t) \equiv & r_{u}^{p}\left(t, t-\tau_{u}^{p}\right) \\
= & {\left[\left(x_{u}(t)-x^{p}\left(t-\tau_{u}^{p}\right)\right)^{2}+\left(y_{u}(t)-y^{p}\left(t-\tau_{u}^{p}\right)\right)^{2}\right.} \\
& \left.+\left(z_{u}(t)-z^{p}\left(t-\tau_{u}^{p}\right)\right)^{2}\right]^{1 / 2} \\
= & \left\|u(t)-s^{p}\left(t-\tau_{u}^{p}\right)\right\|,
\end{aligned}
$$

where $u \equiv\left[x_{u}, y_{u}, z_{u}\right]^{\mathrm{T}}$ and $s^{p} \equiv\left[x^{p}, y^{p}, z^{p}\right]^{\mathrm{T}}$ are the user (unknown) and satellite positions, respectively. Also $n_{s}$ shows the number of the observable satellites. Further in equation $(1), \delta I_{u}^{p}(t)$ and $\delta T_{u}^{p}(t)$ reflect the delay or the advance associated with the transmission of the L1 signal through the ionosphere and the troposphere, respectively. $\delta t_{u}(t)$ and $\delta t^{p}\left(t-\tau_{u}^{p}\right)$ are the clock errors of the receiver $u$ at the time $t$ and the satellite $p$ at the time $t-\tau_{u}^{p} . B_{C A, u}^{p}(t)$ contains anomalous measurements in the usual observed positioning data consisting of L1 pseudoranges based on $\mathrm{C} / \mathrm{A}$ codes. Further in equation (2), the equation consists of the derivative terms of the corresponding elements in equation (1). $b_{D L 1, u}^{p}(t)$ also contains anomalous measurements based on Doppler shift frequency. $e_{C A, u}^{p}(t), \varepsilon_{D L 1, u}^{p}(t)$ denote observation errors.

Equation (3) contains the satellite orbital errors. The estimated satellite orbits are obtained from the navigation messages which are decoded from the transmitted L1 signal. Let us denote $\hat{s}^{p}$ as the estimated position of the satellite $s^{p}$ at the time $t-\tau_{u}^{p}$.

We use the following relations of the derivatives

$$
\begin{array}{ll}
\frac{\partial r_{u}^{p}}{\partial x_{u}}=\frac{\left(x_{u}-x^{p}\right)}{r_{u}^{p}}, & \frac{\partial r_{u}^{p}}{\partial y_{u}}=\frac{\left(y_{u}-y^{p}\right)}{r_{u}^{p}}, \\
\frac{\partial r_{u}^{p}}{\partial z_{u}}=\frac{\left(z_{u}-z^{p}\right)}{r_{u}^{p}}, & \left(p=1,2, \ldots, n_{s}\right),
\end{array}
$$

and

$$
\begin{array}{ll}
\frac{\partial r_{u}^{p}}{\partial x^{p}}=-\frac{\left(x_{u}-x^{p}\right)}{r_{u}^{p}}, & \frac{\partial r_{u}^{p}}{\partial y^{p}}=-\frac{\left(y_{u}-y^{p}\right)}{r_{u}^{p}}, \\
\frac{\partial r_{u}^{p}}{\partial z^{p}}=-\frac{\left(z_{u}-z^{p}\right)}{r_{u}^{p}}, & \left(p=1,2, \ldots, n_{s}\right) .
\end{array}
$$

Then we have the relation:

$$
\frac{\partial r_{u}^{p}}{\partial u}=-\frac{\partial r_{u}^{p}}{\partial s^{p}}
$$

Thus the 1st order Taylor series approximation of equation (3) around the previous estimated value $u=$ $\hat{u}^{(j)}$ and $s^{p}=\hat{s}^{p}$ is given by

$$
\begin{aligned}
r_{u}^{p} & \cong r_{\hat{u}^{(j)}}^{\hat{p}}+g_{\hat{u}^{(j)}}^{\hat{p}}\left[u-s^{p}-\left(\hat{u}^{(j)}-\hat{s}^{p}\right)\right] \\
& =\left\|\hat{u}^{(j)}-\hat{s}^{p}\right\|+\frac{\left(\hat{u}^{(j)}-\hat{s}^{p}\right)^{\mathrm{T}}}{\left\|\hat{u}^{(j)}-\hat{s}^{p}\right\|}\left[u-s^{p}-\left(\hat{u}^{(j)}-\hat{s}^{p}\right)\right] \\
& =\frac{\left(\hat{u}^{(j)}-\hat{s}^{p}\right)^{\mathrm{T}}}{\left\|\hat{u}^{(j)}-\hat{s}^{p}\right\|}\left(u-s^{p}\right),
\end{aligned}
$$

for $p=1,2, \ldots, n_{s}$, where

$$
g_{\hat{u}^{(j)}}^{\hat{p}} \equiv\left[\frac{\partial r_{u}^{p}}{\partial u}\right]_{u=\hat{u}^{(j)}, s^{p}=\hat{s}^{p}}^{\mathrm{T}}=\frac{\left(\hat{u}^{(j)}-\hat{s}^{p}\right)^{\mathrm{T}}}{\left\|\hat{u}^{(j)}-\hat{s}^{p}\right\|} .
$$

From equation (1), therefore, we have the approximation:

$$
\begin{aligned}
\rho_{C A, u}^{p} \cong & g_{\hat{u}^{(j)}}^{\hat{p}}\left(u-s^{p}\right) \\
& +\delta I_{u}^{p}+\delta T_{u}^{p}+c\left(\delta t_{u}-\delta t^{p}\right) \\
& +B_{C A, u}^{p}+e_{C A, u}^{p}
\end{aligned}
$$

Also from equation (2), we have the approximation:

$$
\begin{aligned}
\dot{\rho}_{D L 1, u}^{p}= & \lambda_{1} D_{L 1, u}^{p} \\
\cong & g_{\hat{u}^{(j)}}^{\hat{p}}\left(\dot{u}-\dot{s}^{p}\right) \\
& +\delta \dot{I}_{u}^{p}+\delta \dot{T}_{u}^{p}+c\left(\delta \dot{t}_{u}-\delta \dot{t}^{p}\right) \\
& +b_{D L 1, u}^{p}+\varepsilon_{D L 1, u}^{p} .
\end{aligned}
$$

Define the $n_{s} \times 3$ matrix:

$$
G_{\hat{u}^{(j)}}^{\hat{s}} \equiv\left[\begin{array}{c}
g_{\hat{u}^{(j)}}^{\hat{1}} \\
g_{\hat{u}^{(j)}}^{2} \\
\vdots \\
g_{\hat{u}^{(j)}}^{\hat{n}_{s}}
\end{array}\right],
$$

namely,

$$
G_{\hat{u}^{(j)}}^{\hat{s}}=\left[\begin{array}{ccc}
\frac{\partial r_{\hat{u}^{(j)}}^{\hat{1}}}{\partial \hat{x}_{u}^{(j)}} & \frac{\partial r_{\hat{u}^{(j)}}^{\hat{1}}}{\partial \hat{y}_{u}^{(j)}} & \frac{\partial r_{\hat{u}^{(j)}}^{\hat{1}}}{\partial \hat{z}_{u}^{(j)}} \\
\frac{\partial r_{\hat{u}^{(j)}}^{\hat{2}}}{\partial \hat{x}_{u}^{(j)}} & \frac{\partial r_{\hat{u}^{(j)}}^{\hat{2}}}{\partial \hat{y}_{u}^{(j)}} & \frac{\partial r_{\hat{u}^{(j)}}^{\hat{2}}}{\partial \hat{z}_{u}^{(j)}} \\
\vdots & \vdots & \vdots \\
\frac{\partial r_{\hat{u}_{s}^{(j)}}^{\hat{n}_{s}}}{\partial \hat{x}_{u}^{(j)}} & \frac{\partial r_{\hat{u}_{s}^{(j)}}^{\hat{n}_{s}}}{\partial \hat{y}_{u}^{(j)}} & \frac{\partial r_{\hat{u}_{s}^{(j)}}^{\hat{n}_{s}}}{\partial \hat{z}_{u}^{(j)}}
\end{array}\right] .
$$

Now let us discuss the satellite's as well as receiver's hardware delays $\delta b_{C A}^{p}$, and $\delta b_{C A, u}$. It was pointed out in [15] that the magnitude of the satellite's hardware 
delay bias is usually in the range of (several nanosecond $\times c$ ) while the receiver's hardware biases could exceed (10 nanoseconds $\times c$ ). Therefore, we assume that the satellite's hardware delay biases are negligible, and the receiver's biases are negligible for the positioning without carrier-phase observables. $B_{C A, u}^{p}$ and $b_{D L 1, u}^{p}$ are zero and negligible for the normal positioning without anomalous observables. $\delta \dot{I}_{u}^{p}(t)$ and $\delta \dot{T}_{u}^{p}(t)$ which are the delay changes for a short time are very small and negligible.

Equations (9) and (10) are denoted for the observables of L1-CA code pseudoranges and Doppler shifts. The equations can be applied for L2-CA, L1PY, L2-PY and other kinds of observables for multifrequencies and multi-GNSS navigation signals.

Here, we define the vectors:

$$
\begin{aligned}
\rho_{C A, u}^{s} \equiv\left[\begin{array}{c}
\rho_{C A, u}^{1} \\
\vdots \\
\rho_{C A, u}^{n_{s}}
\end{array}\right], \quad D_{L 1, u}^{s} \equiv\left[\begin{array}{c}
D_{L 1, u}^{1} \\
\vdots \\
D_{L 1, u}^{n_{s}}
\end{array}\right], \\
c \delta t^{s} \equiv\left[\begin{array}{c}
c \delta t^{1} \\
\vdots \\
c \delta t^{n_{s}}
\end{array}\right], \\
s \equiv\left[\begin{array}{c}
s^{1} \\
\vdots \\
s^{n_{s}}
\end{array}\right], \quad \dot{s} \equiv\left[\begin{array}{c}
\dot{s}^{1} \\
\vdots \\
\dot{s}^{n_{s}}
\end{array}\right], \\
\delta I_{u} \equiv\left[\begin{array}{c}
\delta I_{u}^{1} \\
\vdots \\
\delta I_{u}^{n_{s}}
\end{array}\right], \quad \delta T_{u} \equiv\left[\begin{array}{c}
\delta T_{u}^{1} \\
\vdots \\
\delta T_{u}^{n_{s}}
\end{array}\right], \\
e_{C A, u} \equiv\left[\begin{array}{c}
e_{C A, u}^{1} \\
\vdots \\
e_{C A, u}^{n_{s}}
\end{array}\right], \quad \varepsilon_{D L 1, u} \equiv\left[\begin{array}{c}
\varepsilon_{D L 1, u}^{1} \\
\vdots \\
\varepsilon_{D L 1, u}^{n_{s}}
\end{array}\right] .
\end{aligned}
$$

Furthermore, from equation (12), we define a block diagonal matrix with the size $\left(n_{s} \times 3 n_{s}\right)$ :

$$
G_{D, \hat{u}^{(j)}}^{\hat{s}} \equiv\left[\begin{array}{ccccc}
g_{\hat{u}^{(j)}}^{\hat{1}} & O & O & \cdots & O \\
O & g_{\hat{u}^{(j)}}^{\hat{2}} & O & \cdots & O \\
\vdots & & \ddots & & \vdots \\
\vdots & & & \ddots & O \\
O & \cdots & \cdots & O & g_{\hat{u}_{s}^{(j)}}^{\hat{n}_{s}}
\end{array}\right]
$$

Then from equations (9)-(10), we have the following vector regression equation:

$$
y_{u}^{s}=H_{\hat{u}^{(j)}}^{\hat{s}} \theta_{u}+v_{u},
$$

where

$$
\begin{aligned}
& y_{u}^{s} \equiv\left[\begin{array}{c}
\rho_{C A, u}^{s} \\
\lambda_{1} D_{L 1, u}^{s}
\end{array}\right], \quad \theta_{u} \equiv\left[\begin{array}{c}
u \\
\dot{u} \\
c \delta t_{u} \\
\dot{\delta} t_{u} \\
c \delta t^{s} \\
s \\
\dot{s} \\
\delta I_{u} \\
\delta T_{u}
\end{array}\right]
\end{aligned}
$$

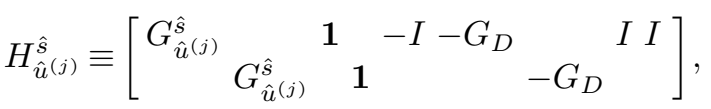

$$
\begin{aligned}
& v_{u} \equiv\left[e_{C A, u}^{\mathrm{T}}, \lambda_{1}\left(\varepsilon_{D L 1, u}^{p}\right)^{\mathrm{T}}\right]^{\mathrm{T}},
\end{aligned}
$$

and $I$ denote the $n_{s} \times n_{s}$ identity matrix and $\mathbf{1} \equiv$ $[1,1, \cdots, 1]^{\mathrm{T}}: n_{s} \times 1$ vector.

Now we assume that the information of the satellite position $s$, the time derivative of the satellite position $\dot{s}$, the satellite clock error $c \delta t^{s}$ as well as the delay or the advance due to the ionospheric and tropospheric effects, $\delta I_{u}^{\mathrm{T}}$ and $\delta T_{u}^{\mathrm{T}}$ are provided with uncertainties as follows:

$$
\begin{aligned}
\hat{s} & =s+e_{s}, \\
\hat{\dot{s}} & =\dot{s}+e_{\dot{s}}, \\
c \hat{\delta} t^{s} & =c \delta t^{s}+e_{\delta t^{s}},
\end{aligned}
$$

and

$$
\begin{aligned}
& \hat{\delta I_{u}}=\delta I_{u}+e_{\delta I_{u}}, \\
& \delta \hat{T}_{u}=\delta T_{u}+e_{\delta T_{u}},
\end{aligned}
$$

where $e_{s}, \cdots, e_{\delta T_{u}}$ are white Gaussian noises denoting uncertainty of the information.

From equations (17)-(21), we have

$$
\begin{aligned}
s & =\hat{s}-e_{s}, \\
\dot{s} & =\hat{\dot{s}}-e_{\dot{s}}, \\
c \delta t^{s} & =c \hat{\delta t^{s}}-e_{\delta t^{s}}, \\
\delta I_{u} & =\hat{\delta I_{u}}-e_{\delta I_{u}}, \\
\delta T_{u} & =\hat{\delta T_{u}}-e_{\delta T_{u}} .
\end{aligned}
$$

Substituting the above equations (22)-(26) into the GR equation (15), we have

$$
y_{u} \equiv\left[\begin{array}{c}
y_{C A, \hat{u}^{(j)}}^{\hat{s}} \\
y_{D L 1, \hat{u}^{(j)}}^{\hat{s}}
\end{array}\right]=C_{\hat{u}^{(j)}}^{\hat{s}}\left[\begin{array}{c}
u \\
\dot{u} \\
c \delta t_{u} \\
c \dot{\delta} t_{u}
\end{array}\right]+v
$$

where

$$
\begin{aligned}
y_{C A, \hat{u}^{(j)}}^{\hat{s}} \equiv & \rho_{C A, u}^{s}+G_{D, \hat{u}^{(j)}}^{\hat{s}} \hat{s} \\
& +c \hat{\delta} t^{s}-\hat{\delta I_{u}}-\delta \hat{T} \\
u & , \\
y_{D L 1, \hat{u}^{(j)}}^{\hat{s}} \equiv & \lambda_{2} D_{L 1, u}^{s}+G_{D, \hat{u}^{(j)}}^{\hat{s}},
\end{aligned}
$$




$$
C_{\hat{u}^{(j)}}^{\hat{s}}=\left[\begin{array}{llll}
G_{\hat{u}^{(j)}}^{\hat{s}} & & & \mathbf{1} \\
& G_{\hat{u}^{(j)}}^{\hat{s}} & & \mathbf{1}
\end{array}\right],
$$

and

$$
v=\left[\begin{array}{c}
G_{D, \hat{u}^{(j)}}^{\hat{s}} e_{s}+e_{\delta t^{s}}-e_{\delta I_{u}}-e_{\delta T_{u}}+e_{C A, u} \\
G_{D, \hat{u}^{(j)}}^{\hat{s}} e_{\dot{s}}+\lambda_{1} \varepsilon_{D L 1, u}^{p}
\end{array}\right] .
$$

\section{Kalman Filter Formulation}

Let us derive the Kalman filtering algorithm for positioning based upon the GR equation (27). For this purpose, we derive the so-called state equation as follows.

\section{State equation}

Let us derive the state equation for kinematic positioning. Since the kinematic case requires mathematical models of the moving objects, we often apply one of the dynamical models which are assumed as first-order Markov processes of, the velocity of $u(: v)$, of the acceleration of $u(: a)$ (the so-called Singer's moving model [16]), or of the jerk of $u(: \gamma),[17,18]$, with or without the constraints $[19,20]$.

For the land vehicle such as automobiles, we adopt Singer's model for the east-west (E) coordinate and the north-south $(\mathrm{N})$ coordinate, and a first order markov model of the velocity for the up-down (U) coordinate, in the local frame [1]. Namely, the unknowns are the position, velocity, and horizontal accelerations in ENU coordinate frame. They are related to the corresponding values in WGS-84 coordinate frame through the linear transformation defined by $3 \times 3$ transformation matrix $T_{W}^{L}$ which transforms WGS-84 coordinates into ENU coordinates [7]. Therefore the state vector is defined as follows:

$$
\begin{aligned}
\eta_{L} \equiv & {\left[u_{L}^{\mathrm{T}}, \dot{u}_{L}^{\mathrm{T}}, a_{L}^{\mathrm{T}}, c \delta t_{u}, c \dot{\delta} t_{u}\right]^{\mathrm{T}} } \\
\equiv & {\left[x_{L}, y_{L}, z_{L}, v_{x, L}, v_{y, L}, v_{z, L}, a_{x, L}, a_{y, L},\right.} \\
& \left.\quad c \delta t_{u}, c \dot{\delta} t_{u}\right]^{\mathrm{T}},
\end{aligned}
$$

where $L$ stands for the coordinates in the local frame (or the local-level system (LLS), or east-north-up (ENU) system) $[1,2]$.

Then by the assumption of the Singer's model, namely the accelerations; $a_{x, L}, a_{y, L}$, of $x_{L}, y_{L}$, respectively, are assumed as the first order Markov processes:

$$
\begin{aligned}
& \dot{a}_{x_{L}}(t)=-\alpha_{x} a_{x_{L}}(t)+w_{a_{x}}(t), \\
& \dot{a}_{y_{L}}(t)=-\alpha_{y} a_{y_{L}}(t)+w_{a_{y}}(t) .
\end{aligned}
$$

Also the velocity of $v_{z, L}$ of $z_{L}$ is assumed as a first order Markov process:

$$
\dot{v}_{z_{L}}(t)=-\alpha_{z} v_{z_{L}}(t)+w_{v_{z}}(t) .
$$

Then, we have the following form of the discrete-time state equation [1]:

$$
\eta_{L}(t+1)=A(t) \eta_{L}(t)+w(t)
$$

where $A(t)$ is the $10 \times 10$ known matrix defined from equations (30) and (32), and details are summarized in [7]. The observation equation $y_{L}(t)$ is based on a local position as a origin and derived from the relation between equation (27) and the transformation by $T_{W}^{L}$ as follows:

$$
\begin{aligned}
& y_{L}(t)=C_{L}(t) \eta_{L}(t)+v(t), \\
& C_{L}(t)=\left[\begin{array}{lllll}
G_{\hat{u}^{(j)}}^{\hat{s}}\left(T_{W}^{L}\right)^{T} & & & \\
& G_{\hat{u}^{(j)}}^{\hat{s}}\left(T_{W}^{L}\right)^{T} & \mathbf{0} & & \mathbf{1}
\end{array}\right],
\end{aligned}
$$

where $G_{\hat{u}^{(j)}}^{\hat{s}}\left(T_{W}^{L}\right)^{T}$ is $n_{s} \times 3$ matrix, $\mathbf{0}$ is $n_{s} \times 2$ zero matrix, and 1 is $n_{s} \times 1$ vector.

The positioning algorithm based on the Kalman filter for equations (33) and (34) can be summarized as follows $[1,21]$ :

$$
\begin{aligned}
\hat{\eta}_{L}(t+1 \mid t)= & A(t) \hat{\eta}_{L}(t \mid t), \\
P(t+1 \mid t)= & A(t) P(t \mid t) A^{\mathrm{T}}(t)+Q(t), \\
\nu(t) \equiv & y(t)-C(t) \hat{\eta}_{L}(t \mid t-1), \\
\hat{\eta}_{L}(t \mid t)= & \hat{\eta}_{L}(t \mid t-1)+K(t) \nu(t), \\
P(t \mid t)= & P(t \mid t-1) \quad(36) \\
& -K(t) C(t) P(t \mid t-1), \\
K(t)= & P(t \mid t-1) C^{\mathrm{T}}(t) \\
& {\left[C(t) \times P(t \mid t-1) C^{\mathrm{T}}(t)+R(t)\right] } \\
& \quad\left(: \operatorname{Kalman}^{-1}\right. \\
& \text { Init. Cond.: }\left\{\begin{array}{l}
\hat{\eta}_{L}(0 \mid-1)=\bar{\eta}_{L}(0) \\
P(0 \mid-1)=P(0)
\end{array}\right.
\end{aligned}
$$

where $Q(t)$ is covariance matrix of system noise, and $R(t)$ is covariance matrix of observation noise.

\section{Detection of Anomalous Measurement}

We apply two methods of detecting Doppler observable outliers. First method is the innovation based detection in Kalman filtering (IBD-method), which is a existing method [22] for more accurate positioning by using carrier-phase observables and utilized for detecting cycle slips of RTK positioning [11]. Second method is a novel method and the measurement based detection (MBD-method) which focuses on the difference between $\mathrm{C} / \mathrm{A}$ code pseudoranges and Doppler shift range-rates. The Both methods are based on the chi-squared tests, and applied for Doppler observables of low cost GNSS receivers to keep the vehicle positioning accuracy high.

\subsection{IBD-method}

Since the innovation process in equation (38) is a white Gaussian with 0 mean and covariance matrix $M(t)\left(=\left[M_{i j}(t)\right] ; i, j=1, \cdots, 2 n_{s}\right)$ under the hypothesis $H_{0}$ such that there are no Doppler outliers. $M(t)$ is formulated as follows [12]: 


$$
\begin{aligned}
M(t) & \equiv \mathrm{E}\left[\nu(t) \nu^{\mathrm{T}}(t)\right] \\
& =\left[C(t) P(t \mid t-1) C^{\mathrm{T}}(t)+R(t)\right],
\end{aligned}
$$

where $P(t \mid t-1)$ is the error covariance matrix of $\hat{\eta}_{L}(t \mid t-$ $1)$. Therefore, under hypothesis $H_{0}$, the $j$-th element of innovation vector $\nu$ which is expressed by $\nu_{j}$ would be the normal distribution with zero mean and variance $M_{j j}$. Then $\nu_{j}$ can be normalized as follows:

$$
\begin{aligned}
& \nu_{n, j}(t) \equiv \sqrt{\frac{1}{M_{j j}(t)}} \nu_{j}(t), \quad j=1, \ldots, 2 n_{s} \\
& \mathrm{E}\left[\nu_{n, j}(t)\right]=0, \quad \operatorname{Var}\left[\nu_{n, j}(t)\right]=1 .
\end{aligned}
$$

Based on equation (43), the anomaly of Doppler frequency observables can be detected by monitoring the normalized innovation processes corresponding to the Doppler shift observables, i.e. $\nu_{n, j}$ for $j=$ $n_{s}+1, \ldots, 2 n_{s}$. Each element of $\nu_{n, j}(t)$ is a Gaussian process. Therefore the test statistics $T_{j}(t)$ of the squared value of $\nu_{n, j}(t)$ follows the chi-squared distribution with 1 degree of freedom, namely,

$$
T_{j}(t)=\left(\nu_{n, j}(t)\right)^{2} .
$$

Statistical tests of innovation processes can be easily extended to the tests based on multiple epochs or observables. $\nu_{n, j, k}$ is the $j$-th innovation vector in equation (43) for $k$ consecutive epochs. The consecutive $\nu_{n, j}$ are independent, therefore the mean vector is zero and the covariance matrix $I$,

$$
\begin{aligned}
\nu_{n, j, k}(t) & =\left[\nu_{n, j}(t), \nu_{n, j}(t-1), \ldots, \nu_{n, j}(t-k+1)\right]^{T} \\
& \sim N\left([0,0, \ldots, 0]^{T}, I\right) .
\end{aligned}
$$

Statistics $T_{j, k}(t)$ of the sum of $k$ consecutive $\nu_{n, j}$ follows the chi-squared distribution with $k$ degrees of freedom,

$$
T_{j, k}(t) \equiv\left(\nu_{n, j, k}(t)\right)^{T}\left(\nu_{n, j, k}(t)\right) .
$$

In order to effectively detect the Doppler anomaly, we focus on chi-squared test of each Doppler shift observable. If Doppler outliers occur, the covariance matrix of the innovation process changes. Therefore we formulate two hypotheses such as,

$$
\begin{aligned}
& H_{0} \text { : the anomal outlier does not occur } \\
& H_{1} \text { : the anomal outlier occurs }
\end{aligned}
$$

Our decision rule of accepting or rejecting the hypothesis is as follows; If $T_{j, k}(t)$ is larger than the upper percent point $\chi_{\alpha}^{2}(k)$ whose $\alpha$ is upper probability $(\alpha=0.05)$ and $k$ is degrees of freedom, then the observed data of Doppler shift contain a outlier (accept $\left.H_{1}\right)$.

\subsection{MBD-method}

The difference value $q^{p}(t)$ between the delta-range and the range-rate from the satellite $p$ at the epoch $t$ is formulated as follows:

$$
q^{p}(t)=\left[\rho^{p}(t)-\rho^{p}(t-1)\right]-\dot{\rho}^{p}(t)
$$

$$
\begin{aligned}
= & {\left[B^{p}(t)-B^{p}(t-1)-b^{p}(t)\right] } \\
& +\left[e^{p}(t)-e^{p}(t-1)-\varepsilon^{p}(t)\right] \\
= & \mu^{p}(t)+d^{p}(t), \\
\mu^{p}(t)= & {\left[B^{p}(t)-B^{p}(t-1)-b^{p}(t)\right], } \\
d^{p}(t)= & {\left[e^{p}(t)-e^{p}(t-1)-\varepsilon^{p}(t)\right], }
\end{aligned}
$$

where in order to simplify the expression, super and sub scripts are dropped here except the super script " $p$ " which indicates the satellite. The difference of geometric distance $r^{p}(t)-r^{p}(t-1)$ in equation (1) and the velocity $\dot{r}^{p}(t)$ in equation (2) are almost the same value and canceled, and the values related to $\delta I^{p}, \delta T^{p}$, $\delta t_{u}$, and $\delta t^{p}$ are also canceled. The bias $\mu^{p}(t)$ based on anomalous measurements $B^{p}, b^{p}$ and the noise $d^{p}(t)$ based on the measurement noises $e^{p}, \varepsilon^{p}$ are left. When there are no anomalous measurements in the both observables of $\mathrm{C} / \mathrm{A}$ code pseudoranges and Doppler shifts, the $q^{p}(t)$ is nearly zero, therefore $\mu^{p}(t)$ is zero, and $q^{p}(t)=d^{p}(t)$.

The noises $e^{p}, \varepsilon^{p}$ are independent white Gaussian with zero mean, and their variance are supposed to be $\sigma_{\rho}^{2}, \sigma_{D}^{2}$, respectively. The mean and variance of $d^{p}(t)$ and $q^{p}(t)$ measured at single epoch are as follows:

$$
\begin{aligned}
& e^{p}(t) \sim N\left(0, \sigma_{\rho}^{2}\right), \varepsilon^{p}(t) \sim N\left(0, \sigma_{D}^{2}\right), \\
& d^{p}(t) \sim N\left(0,2 \sigma_{\rho}^{2}+\sigma_{D}^{2}\right), \\
& q^{p}(t) \sim N\left(\mu^{p}(t), 2 \sigma_{\rho}^{2}+\sigma_{D}^{2}\right) .
\end{aligned}
$$

The variance matrix of the noise $d_{t, j}^{p}$ between two different epochs $t, j$ is as follows:

$$
\begin{aligned}
\mathrm{E}\left[\begin{array}{l}
d^{p}(t) \\
d^{p}(j)
\end{array}\right]\left[\begin{array}{l}
d^{p}(t) \\
d^{p}(j)
\end{array}\right]^{T} & =\mathrm{E}\left[\begin{array}{cc}
d^{p}(t)^{T} d^{p}(t) & d^{p}(t)^{T} d^{p}(j) \\
d^{p}(j)^{T} d^{p}(t) & d^{p}(j)^{T} d^{p}(j)
\end{array}\right] \\
& =\left[\begin{array}{cc}
2 \sigma_{\rho}^{2}+\sigma_{D}^{2} & -\sigma_{\rho}^{2} \\
-\sigma_{\rho}^{2} & 2 \sigma_{\rho}^{2}+\sigma_{D}^{2}
\end{array}\right] \\
& =\left[\begin{array}{cc}
2 \sigma_{\rho}^{2}+\sigma_{D}^{2} & (j=t \pm 1) \\
0 & 2 \sigma_{\rho}^{2}+\sigma_{D}^{2}
\end{array}\right] \\
& (j \neq t \pm 1)
\end{aligned}
$$

where the covariance value of two consecutive epochs is $-\sigma_{\rho}^{2}$ when $j=t \pm 1$, and zero when $j \neq t \pm 1$.

$d_{k}^{p}$ is the measurement noise vector in equation (50) which uses $k$ consecutive epochs. The mean vector is zero and the covariance matrix $\Sigma$ is not a diagonal matrix, namely,

$$
\begin{aligned}
d_{k}^{p}(t) & =\left[d^{p}(t), d^{p}(t-1), \ldots, d^{p}(t-k+1)\right]^{T} \\
& \sim N\left([0,0, \ldots, 0]^{T}, \Sigma\right) .
\end{aligned}
$$

The zero covariance matrix $\Sigma$ is decomposed to generate the unitary matrix $U$ as follows:

$$
\Sigma=U^{-1} U^{-T} \text {. }
$$

where the zero covariance matrix of the normalized noise vector $d_{n, k}^{p}=U d_{k}^{p}$ becomes identity matrix.

$q_{k}^{p}$ is the measurement difference vector in equation (48) which uses $k$ consecutive epochs. The normalized vector $q_{n, k}^{p}$ is generated by the unitary matrix 
$U$ in the same way. The statistics $T_{k}^{p}(t)$ of the sum of squared $q_{n, k}^{p}$ follows the chi-squared distribution with $k$ degree of freedom, namely,

$$
\begin{aligned}
q_{k}^{p}(t) & =\left[q^{p}(t), q^{p}(t-1), \ldots, q^{p}(t-k+1)\right]^{T}, \\
q_{n, k}^{p}(t) & \equiv U q_{k}^{p}(t), \\
T_{k}^{p}(t) & \equiv\left(q_{n, k}^{p}(t)\right)^{T}\left(q_{n, k}^{p}(t)\right) .
\end{aligned}
$$

If Doppler outliers occur, the measurement difference vector $q^{p}(t)$ changes. Therefore we formulate two hypotheses such as,

$$
\begin{aligned}
& H_{0}(\text { normal }): T_{k}^{p}<\chi_{\alpha}^{2}(k) \\
& H_{\alpha}(\text { abnormal }): T_{k}^{p} \geq \chi_{\alpha}^{2}(k)
\end{aligned}
$$

Our decision rule of accepting or rejecting the hypothesis is as follows; If $T_{k}^{p}(t)$ is larger than the upper percent point $\chi_{\alpha}^{2}(k)$ whose $\alpha$ is upper probability $(\alpha=0.05)$ and $k$ is degrees of freedom, then the observed data of Doppler shift contain a outlier (accept $H_{\alpha}$ ). The $\alpha$ shows the probability of false alarm $P_{F A}$.

If Doppler observables have a outlier, the statistics $T_{k}^{p}(t)$ follows the non-central chi-squared distribution with non-central parameter $\lambda$ as follows:

$$
\begin{aligned}
H_{1} \text { (abnormal) }: T_{k}^{p} & \approx \chi^{2}(k, \lambda) \\
& \left(\lambda \equiv\left(U \mu^{p}\right)^{T}\left(U \mu^{p}\right)\right)
\end{aligned}
$$

and the detectable minimum bias $T_{\text {bias }}$ of Doppler observables is derived from the $\lambda$ as follows:

$$
T_{\text {bias }}=\sigma_{D} \sqrt{\lambda}
$$

and when the probability of missed detection $P_{M D}$ is defined as the lower probability $\beta$ of non-central $\chi^{2}(k, \lambda)$ distribution, and the lower percent point $\chi_{\beta}^{2}(k, \lambda)$ is equal to the upper percent point $\chi_{\alpha}^{2}(k)$ of normal $\chi^{2}(k)$ distribution, the $\lambda$ is decided and the $T_{\text {bias }}$ is derived from equation (60). The protection levels of RAIM(Receiver Autonomous Integrity Monitoring) are defined by $P_{F A}, P_{M D}$, and $T_{\text {bias }}$ (pp. 199-202 in [1]).

\section{Correction of Doppler Biases}

We focus on the correction of Doppler outliers $b_{D L 1}^{p}$ in equation (2), because we had a positioning error data of the real test drive whose Doppler-aided positioning was affected by a Doppler bias. The biased Doppler shift observables are detected by the IBD or MBD-methods, and then the bias impacts are removed by the following two methods which are Doppler bias exclusion, or Doppler bias estimation.

In the case of Doppler bias exclusion, if the Doppler bias is detected in the $j$-th satellite, then the related observable, i.e. the $j$-th component of $y_{D L 1, \hat{u}}^{\hat{s}}$ and the related row of observation matrix $C(t)$ in equations (38-41) are excluded. The observation noises related to $y_{D L 1, \hat{u}}^{\hat{s}}$ are similarly excluded from the covariance matrix $R(t)$ in equation (41). For example, if $j=2$, the 2nd Doppler observable and the related row components with brackets are excluded as follows:

$$
\left[\begin{array}{c}
y_{C A, \hat{u}}^{\hat{1}} \\
\vdots \\
y_{C A, \hat{u}}^{\hat{n}_{s}} \\
y_{D L 1, \hat{u}}^{\hat{1}} \\
\left(y_{D L 1, \hat{u}}^{\hat{2}}\right) \\
\vdots \\
y_{D L 2, \hat{u}}^{\hat{n}_{s}}
\end{array}\right]=\left[\begin{array}{cccc}
G_{\hat{u}}^{\hat{1}} & 0 & 1 & 0 \\
& \vdots & & \\
G_{\hat{u}}^{\hat{n}_{s}} & 0 & 1 & 0 \\
0 & G_{\hat{u}}^{\hat{1}} & 0 & 1 \\
(0) & \left(G_{\hat{u}}^{2}\right) & (0) & (1) \\
\vdots & & \\
0 & G_{\hat{u}}^{\hat{n}_{s}} & 0 & 1
\end{array}\right]\left[\begin{array}{c}
u \\
\dot{u} \\
c \delta t_{u} \\
c \dot{\delta} t_{u}
\end{array}\right]+v .
$$

In the case of Doppler bias estimation, if the Doppler bias is detected in the $j$-th satellite, then Doppler bias will be augmented as unknown parameter $b_{D L 1}^{j}$ for the $j$-th component of $y_{D L 1, \hat{u}}^{\hat{s}}$ to the state vector $\eta_{L}$ in equation (36), and the related column is added to the last column of observation matrix $C(t)$ in equations (38-41). The initial values of the unknown parameters $b_{D L 1}^{j}$ are set to the difference values between Doppler range-rates and code delta-ranges. The initial variance 0.1 related to $b_{D L 1}^{j}$ are added into estimation error covariance matrix $P(t \mid t)$ in equation (37). The model of Doppler bias $b_{D L 1}^{j}$ is defined by the state transition matrix $A(t)$ in equations (36) and (37) is as follows:

$$
b_{D L 1}^{j}(t+1)=b_{D L 1}^{j}(t)+w_{b}(t) .
$$

The system noise 0.01 related to the $b_{D L 1}^{j}$ are added into covariance matrix $Q(t)$ in equation (37).

For example, if $j=2$, the unknown parameter for 2nd Doppler observable and the related column with brackets are added into the state vector and the observation matrix, respectively as follows:

$$
\left[\begin{array}{c}
y_{C A, \hat{u}}^{\hat{1}} \\
\vdots \\
y_{C A, \hat{u}}^{\hat{n}_{s}} \\
y_{D L 1, \hat{u}}^{\hat{1}} \\
y_{D L 1, \hat{u}}^{\hat{2}} \\
\vdots \\
y_{D L 1, \hat{u}}^{\hat{n}_{s}}
\end{array}\right]=\left[\begin{array}{ccccc}
G_{\hat{u}}^{\hat{1}} & 0 & 1 & 0 & (0) \\
& \vdots & & & \\
G_{\hat{u}}^{\hat{n}_{s}} & 0 & 1 & 0 & (0) \\
0 & G_{\hat{u}}^{\hat{1}} & 0 & 1 & (0) \\
0 & G_{\hat{u}}^{2} & 0 & 1 & (1) \\
& \vdots & & & \\
0 & G_{\hat{u}}^{\hat{n}_{s}} & 0 & 1 & (0)
\end{array}\right]\left[\begin{array}{c}
u \\
\dot{u} \\
c \delta t_{u} \\
\dot{\delta \delta} t_{u} \\
\left(b_{D L 1}^{2}\right)
\end{array}\right]+v .
$$

\section{Experimental Results}

\subsection{Doppler Bias Sample}

We had a vehicle positioning error in real test drives under open sky environment. The receiver was u-blox NEO-7N (measurable only one frequency L1), and the vehicle with it moved in the streets in Maryland, US, on April 15, 2014, from 17:14' 41 to 17:32' 05 UTC. Fig. 2 shows the Doppler range-rate bias of SBAS (Satellite-Based Augmentation System) satellite (black line) which causes the error. The bias is extracted by subtracting the delta-ranges form the range-rates of the satellite, and has the rectangular shape whose length is from epoch 60 to 280 and size is about $10[\mathrm{~m} / \mathrm{s}]$. 


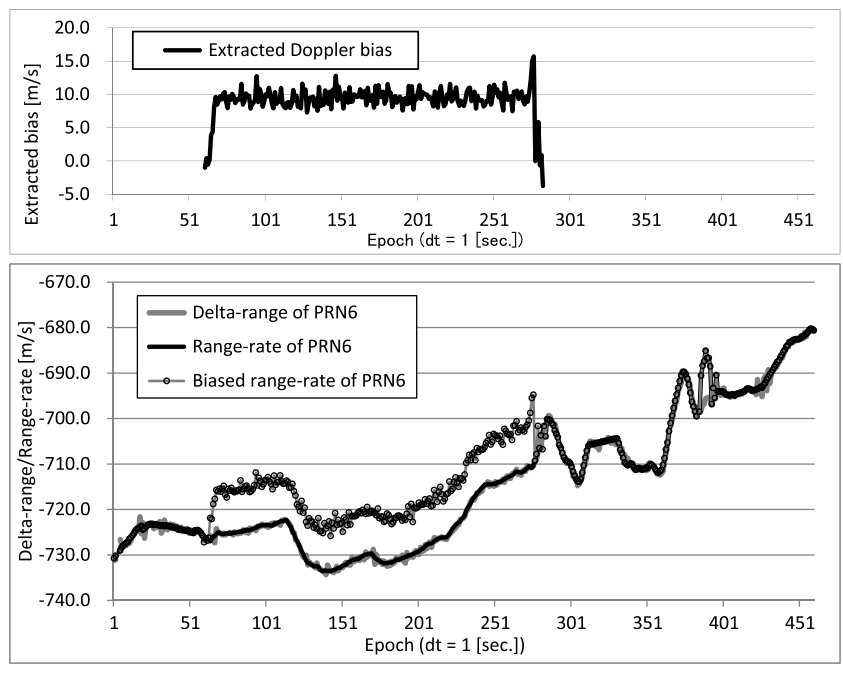

Fig. 2 Extracted Doppler bias and experimental sample

In order to reproduce the error by our GR model which is the GPS and Doppler-based Kalman filter positioning, the extracted Doppler range-rate bias is injected to the original range-rate of PRN6 as a experimental sample (line with circle). The delta-ranges of PRN6 (gray line) are almost the same values as the original range-rates of PRN6 (black line) because of open sky environment. The PRN6 is the closest GPS satellite to the SBAS satellite. We confirmed that Doppler dominant positioning causes positioning errors even under open sky environments due to the Doppler bias sample [8]. In general, Doppler shift frequency observable is derived from the change of the frequency of the received signal caused by the relative motion of the GNSS satellite and the receiver. The GNSS signals are affected by not only the motions but also the fluctuation on the travel path, and various geomorphic environments such as scintillation, interference, multipath, and so on.

\subsection{Detection and Correction Results}

The experiment of Kalman filter-based positioning has been carried out to detect and correct the Doppler biases. Table 1 shows the conditions of experiments (a)-(e) combined of the IBD or MBD-methods, the exclusion or estimation correction methods, and a square or a slope bias wave. C/A pseudorange and Doppler shift noise variances are supposed to be $5^{2}[\mathrm{~m}]$ and $0.5^{2}[\mathrm{~m} / \mathrm{s}]$ for all experiments. The variances are decided by the one-sigma standard deviation error model of pseudoranges [3], and the measurement analysis of the fixed point positioning of NEO-7N receiver under open sky environments [8]. MBD-method also uses $\sigma_{\rho}^{2}=5[\mathrm{~m}]$ and $\sigma_{D}^{2}=0.5[\mathrm{~m} / \mathrm{s}]$ as the noise variances for pseudoranges and range-rates, respectively.

The IBD or MBD-methods are applied for experiments (a),(b),(d), or experiments (c),(e), respectively. The bias exclusion or estimation methods are applied for experiments (a),(c),(d),(e), or experiment (b), respectively. The same upper probability $\alpha=0.05$
Table 1 Experimental conditions

\begin{tabular}{|l||c|c|c|c|c|c|}
\hline & (a) & (b) & (c) & (d) & $(\mathrm{e})$ \\
\hline \hline \multicolumn{1}{|c||}{ C/A pseudorange noise variance } & \multicolumn{5}{|c|}{$5^{2}\left[\mathrm{~m}^{2}\right]$} \\
\hline Doppler shift noise variance & \multicolumn{5}{c|}{$0.5^{2}\left[(\mathrm{~m} / \mathrm{s})^{2}\right]$} \\
\hline \hline IBD and exclusion method & $\mathrm{O}$ & - & - & $\mathrm{O}$ & - \\
\hline IBD and estimation method & - & $\mathrm{O}$ & - & - & - \\
\hline MBD and exclusion method & - & - & $\mathrm{O}$ & - & $\mathrm{O}$ \\
\hline \hline Upper probability $\alpha$ of $\chi^{2}$ tests & \multicolumn{5}{|c|}{0.05} \\
\hline \hline Square bias wave & $\mathrm{O}$ & $\mathrm{O}$ & $\mathrm{O}$ & - & - \\
\hline Slope bias wave & - & - & - & $\mathrm{O}$ & $\mathrm{O}$ \\
\hline
\end{tabular}

of chi-squared tests are used for the both IBD and MBD-methods. The $k$ degrees of freedom for the chisquared tests are decided by the number of consecutive epochs for testing which is window width $k$. The window width is decided to achieve the better detection performance.

The square or slope bias are used for experiments (a),(b),(c), or experiments (d),(e), respectively. The square bias wave is the original extracted bias rangerates, and we prepare for the slope bias to evaluate the detection response according to the bias size. The bias wave is formed by multiplying slope coefficient to the square bias wave.

Fig. 3 shows the comparison of two correction methods. They are experiment (a) with exclusion and experiment (b) with estimation at the same IBDmethod whose window width is 1 epoch.

In experiment (a), during the bias-injected period (black line), the Doppler observable of the target satellite PRN6 which includes the Doppler bias sample is synchronously excluded (blue line), and the exclusion method almost can have no response delays at the start and end points of the bias. However a few satellites which includes PRN6 are excluded (green line) during about 30 percent of the bias-injected period. The number of normal Doppler shift observables which are utilized for positioning are decreased.

In experiment (b), during the bias-injected period, the estimated bias values (yellow line) are almost the same as the extracted bias. Few other satellites' Doppler biases are estimated (green line), because the start condition for the bias estimation is that $H_{1}$ acceptances by Hypothesis testing of IBD-method continue for 3 consecutive epochs. The bias estimations are stopped after several epochs when the estimation value is less than specific threshold level $1.5[\mathrm{~m} / \mathrm{s}]$. Although the start condition can mask spikes of Doppler innovation values and be effective to avoid type-I errors for normal Doppler shifts, the condition causes the response delay of estimation process for several epochs.

Fig. 4 shows the comparison of two detection methods. They are experiments (a),(d) with IBD and experiments (c),(e) with MBD at the same exclusion correction method. The square or slope bias are used for experiments (a),(c), or experiments (d),(e), respectively. 
The IBD-method chooses 1 epoch as the window width, because the statistics $T_{j, k}$ in equation (47) can be more widely affected by spikes of Doppler observables when $k \geq 2$ than $k=1$, and normal Doppler observables are unintentionally excluded for several consecutive epochs. The excess exclusions degrade the detection capability of the IBD-method. While the MBD-method chooses 4 epochs as the window width, because the statistics $T_{k}^{p}$ in equation (59) doesn't surpass the upper percent point even during the biasinjected period when $k=1$. $k=4$ can effectively detect the Doppler bias and keep the smaller response delay of correction processes.

In the comparison between experiments (a) and (c) using square bias (black line), the both detection methods synchronously exclude the target satel-

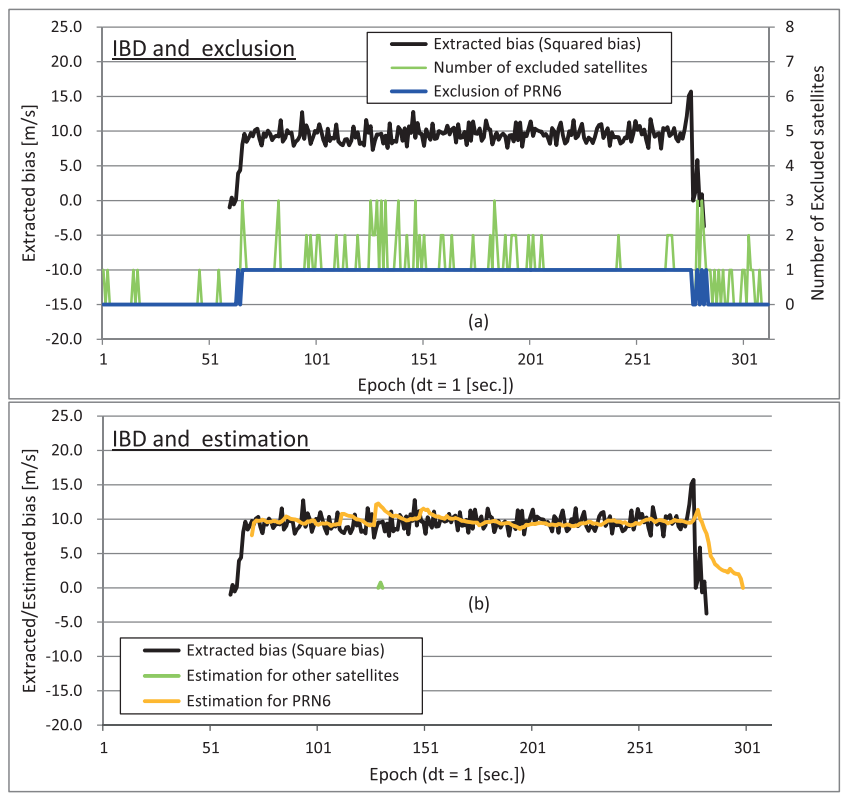

Fig. 3 Doppler bias correction by (a) IBD and exclusion,

(b) IBD and estimation
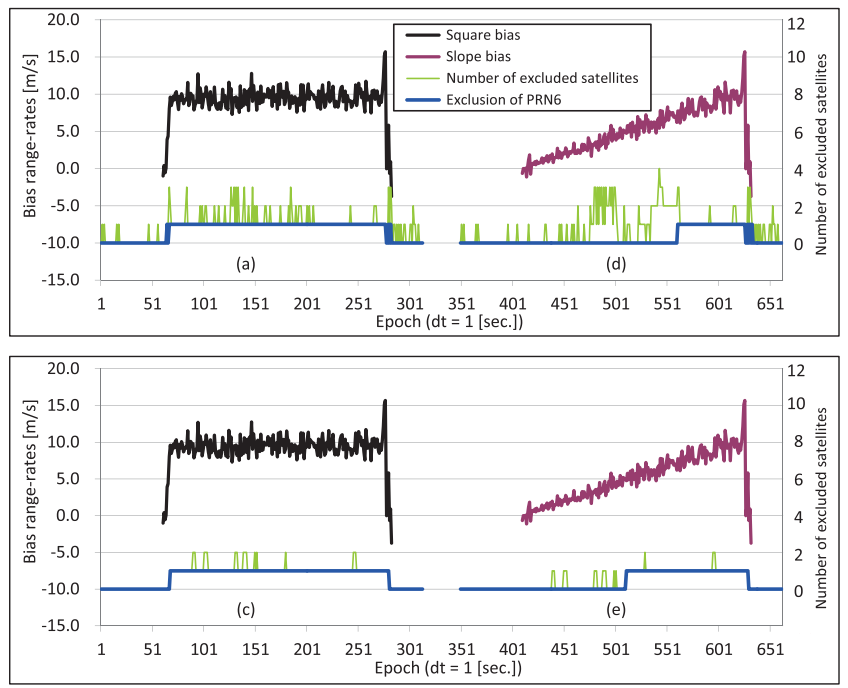

Fig. 4 Square/Slope Doppler bias correction by (a),(d) IBD and exclusion, (c),(e) MBD and exclusion lite PRN6 (blue line) during the bias-injected period. There are no critical response delays by the detection processes at the start and end points of the period. In the comparison between experiments (d) and (e) using slope bias (brown line), the both detection methods have the response delays (blue line). The exclusion of PRN6 is earlier started by experiment (e) than experiment (d). Experiments (c),(e) have less number of excluded other normal satellites (green line) than experiments (a),(d) because of the observables obtained under open sky environments. The MBD-method can effectively detect Doppler biases when $\mathrm{C} / \mathrm{A}$ code pseudorange observables do not have anomalies caused by noises or multipath. Therefore the MBD-method is required to combine with the quality monitoring process of the $\mathrm{C} / \mathrm{A}$ code pseudoranges.

Fig. 5 shows the comparison of the positioning results with no correction (green line) or with three cor-

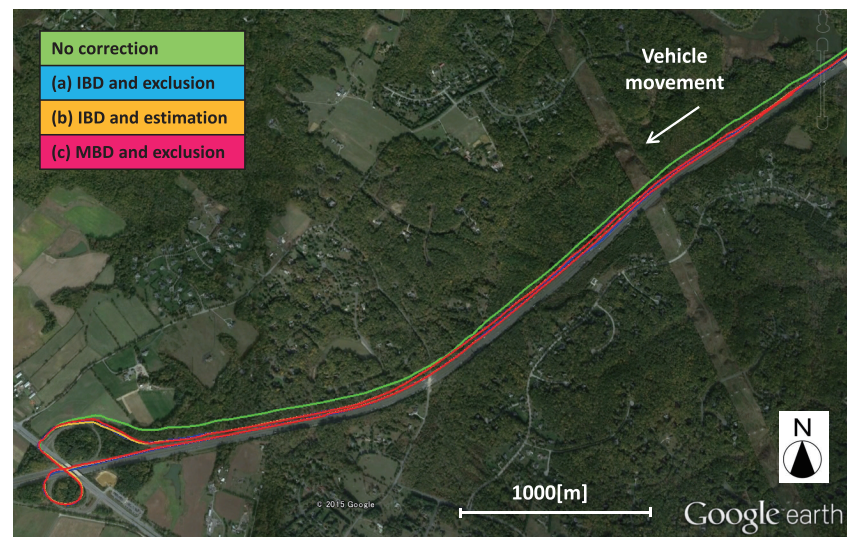

Fig. 5 Vehicle trajectories corrected by (a) IBD and exclusion, (b) IBD and estimation,(c) MBD and exclusion

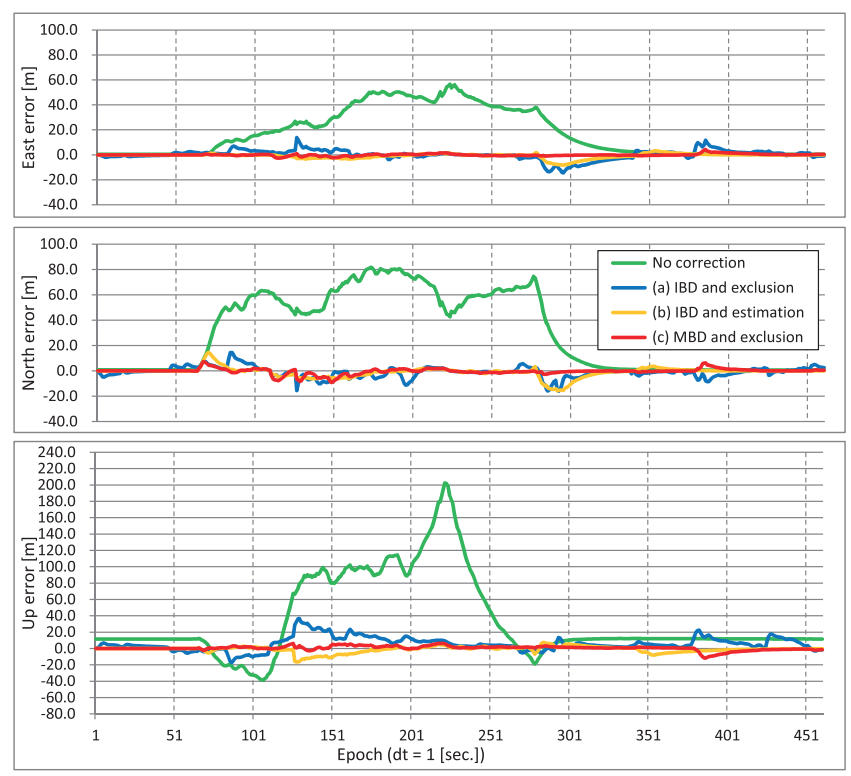

Fig. 6 ENU errors of (a) IBD and exclusion,(b) IBD and estimation, (c) MBD and exclusion 
rections (a),(b),(c) of the square Doppler bias. The gaps between the green trajectory with no correction and the road gradually get wider due to the abnormal vehicle speed vectors caused by the Doppler bias. The deviated green trajectory is corrected by three correction experiments (a),(b),(c), and the corrected trajectories come close to the road.

Fig. 6 shows ENU errors of correction results. The positioning results by original data which include no Doppler bias are regarded as the reference trajectory for evaluating positioning errors. No correction (green line) causes that the ENU errors are more than $20[\mathrm{~m}]$ in the Doppler bias-injected period. Contrarily, three correction experiments result in almost less than 20 [m] errors. Experiment (a),(blue line) results in that there are some small positioning errors even when no Doppler bias injection because of the excess exclusion of normal satellites. Experiment (b), (yellow line) results in that positioning errors are bigger than experiment (a) at the start and end points of the biasinjected period because of some response delays of estimation process. Experiment (c),(red line) results in that positioning errors are smaller than experiments (a) and (b) from the start to the end. The positioning misalignments from the reference positioning are almost less than $10[\mathrm{~m}]$.

\section{Conclusions}

In this paper, a novel MBD-method and the existing IBD-method combined with the exclusion or the estimation correction methods has been proposed for the Doppler bias problems of the low cost single frequency GPS receiver, and been able to remove the impacts of the Doppler bias to keep the vehicle position accuracy high. We have formulated the Doppleraided Kalman filter positioning from the GR models of precise positioning. Experiments of the detection and correction methods have been done by using the real receiver data and artificially simulated the Doppler bias. We have shown that the both IBD and MBD-methods can properly detect the Doppler bias under open sky environments, and the exclusion method can be easier implemented because of the smaller response delays at the start and end points of the bias-injected period than the estimation method.

The window widths of IBD or MBD-methods are decided to achieve better detection performance under the same upper probability of chi-squared tests. We consider that more test samples are needed to verify the window widths of the detection methods. Also we consider that Robust Kalman Filter methods can be applied for GNSS observable outliers at a next step, because it is reported to be effective to reduce the impacts of observable spike noises $[9,10]$. The number of satellite signals for $\mathrm{C} / \mathrm{A}$ code pseudoranges and Doppler shift observables increases more and more by the operation start of multi-frequency and multi-GNSS systems. Our proposal can achieve the selective utilization of the better signals and have the possibility to be effective for more precise positioning.

\section{References}

[1] S. Sugimoto and R. Shibasaki (Eds.): GPS Handbook (in Japanese), Asakura-Shoten (2010)

[2] P. Misra and P. Enge: Global Positioning System Signals, Measurements, and Performance, 2nd Edition, Ganga-Jamuna Press (2006)

[3] B. W. Parkinson and J. J. Spilker Jr. (Eds.): Global Positioning System: Theory and Applications, Vol. I, II, AIAA (1997)

[4] M. E. Cannon: High accuracy GPS semi-kinematic positioning: Modelling and results; Proc. 2nd Int. Tech. Meeting of the Satellite Division of the Institute of Navigation (ION GPS-89), pp. 405-418 (1989)

[5] M. Bahrami and M. Ziebart: Doppler-aided positioning: Improving single-frequency RTK in the urban environment; GPS World, pp. 47-56 (2011)

[6] M. Bahrami and M. Ziebart: A Kalman filter-based Doppler-smoothing of code pseudoranges in GNSSchallenged environments; Proc. 24th Int. Technical Meeting of the Satellite Division of the Institute of Navigation (ION GNSS 2011), pp. 2362-2372 (2011)

[7] A. Chabata, Y. Suzuki, Y. Kubo and S. Sugimoto: RTK-PPP algorithms using GNSS observables from few satellites; Proc. 25th Int. Technical Meeting of the Satellite Division of the Institute of Navigation (ION GNSS 2012), pp. 3696-3707 (2012)

[8] A. Mouri, Y. Kubo and S. Sugimoto: Detection and correction of Doppler biases in Kalman filter-based positioning; Proc. of the 46th ISCIE International Symposium on Stochastic Systems Theory and Its Applications (SSS'14), pp. 156-164 (2014)

[9] T. Nakamizo: Robust estimation (in Japanese); Measurement and Control, Vol. 23, No. 6, pp. 541-549 (1984)

[10] Y. Kaneda, Y. Irizuki and M. Yamakita: Design methods of robust Kalman filter based on statistics and its application (in Japanese); Trans. of ISCIE, Vol. 27, No. 2, pp. 49-58 (2014)

[11] Y. Kubo, K. Sone and S. Sugimoto: Fault detection in carrier phase GPS positioning based on hypotheses testing of innovation processes; Int. J. of Innovative Computing, Information and Control, Vol. 1, No. 3, pp. 461-478 (2005)

[12] M. Kamimura, R. Tomita, T. Nagano, A. Chabata Y. Kubo and S. Sugimoto: Detection of cycle slips and multipath in GNSS RTK precise point positioning; Proc. 24th Int. Technical Meeting of the Satellite Division of the Institute of Navigation (ION GNSS 2011), pp. 1056-1067 (2011)

[13] E. D. Kaplan and C. J. Hegarty (Eds.): Understanding GPS: Principles and Applications 2nd edition, Artech House (2006)

[14] B. Hofmann-Wellenhof, H. Lichtenegger and J. Collins: GPS Theory and Practice 5th edition, Springer (2000)

[15] Y. Gao and Z. Z. Lin: Precise ionosphere modeling using regional GPS network data; J. of Global Positioning Systems, Vol. 1, No. 1, pp. 18-24 (2002)

[16] R. A. Singer: Estimating optimal tracking filter performance for manned maneuvering targets; IEEE 
Trans. Aerospace and Electronic Systems, Vol. AES6, No. 4, pp. 473-483 (1970)

[17] T. Aoki, Y. Shimogaki, T. Ikki, M. Tanikawara, S. Sugimoto, Y. Kubo and K. Fujimoto: Cycle slip detection in kinematic GPS with a jerk model for land vehicles; Int. J. of Innovative Computing, Information and Control, Vol. 5, No. 1, pp. 153-166 (2009)

[18] T. Aoki and S. Sugimoto: Dynamical models for automobile movements; Int. J. of Innovative Computing, Information and Control, Vol. 6, No. 1, pp. 3-14 (2009)

[19] C. Uratani, K. Sone, Y. Muto, S. Maruo and S. Sugimoto: A new method for carrier-phase-based precise point; Proc. 16th Int. Technical Meeting of the Satellite Division of the Institute of Navigation (ION GPS/GNSS 2003), pp. 809-818 (2003)

[20] Y. Muto, Y. Kubo and S. Sugimoto: New dynamical models for kinematic GPS positioning; Proc. 17th Int. Technical Meeting of the Satellite Division of the Institute of Navigation (ION GPS/GNSS 2003), pp. 2519-2528 (2004)

[21] S. Sugimoto and Y. Kubo: Unified methods of point and relative positioning based on GNSS regression equations; Proc. 19th Int. Technical Meeting of the Satellite Division of the Institute of Navigation (ION GNSS 2006), pp. 345-358 (2006)

[22] M. Basseville: Detecting changes in signals and systems; Automatica, Vol. 24, No. 3, pp. 499-501 (1979)

\section{Authors}

\section{Atsushi Mouri (Student Member)}

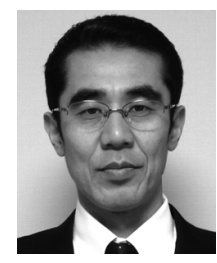

Atsushi Mouri received the B.S. (1987), M.S. (1989) degrees in Electrical Engineering from Osaka Prefecture University, Osaka, Japan. He joined LSI laboratory of Mitsubishi Electric Corp. in 1989, and moved to Information Technology Center in 1993, and presently works for Car Multimedia System Engineering Dept. of Sanda works from 2002. He is presently a Ph.D. student at graduate school of Science and Engineering, Ritsumeikan University from 2014. His research interests include GPS/GNSS signal processing and position information systems.

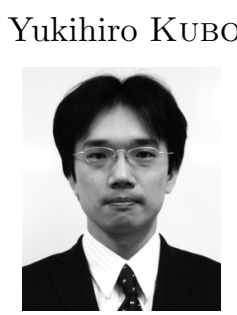

(Member)

Yukihiro Kubo received the B.S. (1997), M.S. (1999) and Ph.D. (2002) degrees in Electrical and electronic engineering from Ritsumeikan University, Shiga and Kyoto, Japan. He worked in the production section of GPS car-navigation systems at Mitsubishi Electric Corp., Sanda Works from 2002 to 2004. He joined Department of Electrical and Electronic Engineering of Ritsumeikan University in 2004, and he is presently a professor. His research interests include GPS/GNSS signal processing and INS/GNSS integration systems.

\section{Sueo Sugimoto (Member)}

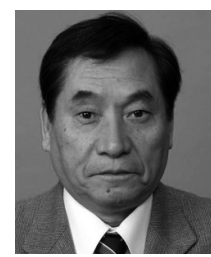

Sueo Sugimoto received the B.S. (1969) and M.S. (1971) degrees in mechanical engineering from the Kyoto Institute of Technology, Kyoto, Japan, and the Ph.D (1974) degree in electrical engineering system science from the Polytechnic Institute of New York (presently, NYU Polytechnic School of Engineering), New York. He is a professor, Department of Electrical and Electronic Engineering at Ritsumeikan University, Shiga and Kyoto, Japan. His research interests include stochastic systems, and statistical image-signal processing in various applications such as GPS/GNSS navigation.

\section{Masaharu OHAshi (Member)}

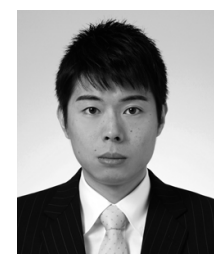

Masaharu Ohashi received the B.S. (2010), M.S. (2012) and Ph.D. (2015) degrees in electrical and electronic engineering from Ritsumeikan University, Shiga, Japan. He joined Department of Electronic System Engineering of The University of Shiga Prefecture in 2015, and he is presently an assistant professor. His research interests include GPS/GNSS signal processing and ionosphere modeling. 